\section{'Pinot noir' and 'Riesling' Grapevines Respond to Water Stress Duration and Soil Water-holding Capacity}

\author{
Andrew G. Reynolds ${ }^{1}$ and Andrew P. Naylor ${ }^{2}$ \\ Agriculture and Agri-Food Canada Research Centre, Summerland, B.C. \\ VOH 1Z0, Canada \\ Additional index words. Vitis vinifera, irrigation deficits, vegetative growth, fruit quality, \\ fruit yield
}

\begin{abstract}
Glasshouse-grown 'Pinot noir' and 'Riesling' grapevines (Vitis vinifera $\mathbf{L}$.) were subjected to one of four water stress durations [no water deficit (control); and water deficits imposed postbloom, lag phase, and veraison] in combination with three soil waterholding capacities $(0 \%, 26 \%$, and $52 \%$ gravel, by volume). Vines subjected to increasing water stress duration had less cumulative lateral shoot length and lower shoot count, leaf size, and berry weights than those not stressed. Soluble solids concentration (SSC) during maturation and $\mathrm{pH}$ at harvest also increased with increasing water stress duration, but titratable acidity was not affected. Transpiration and stomatal conductance also were reduced with increased water stress duration, but soil water increased, reflecting the larger leaf surface on vines with veraison-imposed deficits. Reducing water-holding capacity (by increasing the percentage of gravel in the soil) tended to increase berry weight and SSC but reduced lateral shoot growth. The $52 \%$ gravel treatments increased transpiration rate and stomatal conductance for 'Riesling' but reduced them slightly in 'Pinot noir'. Percentage of soil moisture was reduced linearly with reduced water-holding capacity. These results indicate that early irrigation deficits may advance fruit maturity of wine grapes with concomitant reductions in vegetative growth. Differential responses of these cultivars to soil water-holding capacity also should help to identify suitable wine grape cultivars as the wine grape industry expands into areas with low water-holding capacity soils.
\end{abstract}

Vigorous grapevines produce dense, shaded canopies that may reduce wine grape quality (Smart et al., 1985). A mild water stress imposed through irrigation deficits (Matthews and Anderson 1988, 1989; Matthews et al., 1987; Trought and Naylor, 1988) may reduce vine vigor and competition for carbohydrates by the growing tips, and it may increase the quality of the fruit and wine produced. As the wine grape industry expands in the Pacific Northwest at a time when water shortages are becoming critical, more definitive information is needed on the response of Vitis vinifera to imposed irrigation deficits and lowmoisture-holding soils. The British Columbia wine industry, in particular, is rapidly expanding its plantings into the arid, sandy sites of the southern Okanagan Valley. The wine industry also is becoming increasingly competitive, and there is anecdotal evidence that reducing irrigation may improve wine quality.

Received for publication 25 Feb. 1994. Accepted for publication 1 July 1994. Contribution no. 853. Gratitude is extended to Doug Wardle for technical assistance. The cost of publishing this paper was defrayed in part by the payment of page charges. Under postal regulations, this paper therefore must be hereby marked advertisement solely to indicate this fact.

${ }^{1}$ Research Scientist, Pomology and Viticulture Section.

${ }^{2}$ Current address: HortResearch, Marlborough Research Center, Blenheim, New Zealand.
We are not aware of any research that has addressed the interaction between water stress duration and soil water-holding capacity on wine grape response. Our experiments were conducted to investigate the response of glasshouse-grown 'Pinot noir' and 'Riesling' grapevines to different water stress durations and soils of various water-holding capacities and to make inferences on their relative effects on vine water status. Preliminary results of a related field study have been reported by Naylor et al. (1992).

\section{Materials and Methods}

Between Apr. and Oct. 1991, the effect of water stress duration on 'Pinot noir' and 'Riesling' grapevines was investigated in a glasshouse at the Agriculture Canada Research Station, Summerland, British Columbia, Canada. One-year-old, own-rooted vines were transplanted 2 Apr. 1991 into 20-liter pots with a predetermined proportion of sandy loam soil and gravel. Four water stress duration treatments (irrigation deficits) $\times$ three soil water-holding capacities (gravel : soil ratios) were arranged in a randomized complete block with a factorial treatment arrangement. In total, there were four and six 12-vine blocks for 'Pinot noir' and 'Riesling', respectively. Each block contained single-vine treatment replications. Irrigation deficits were no deficit (control), postbloom, lag phase, and veraison; the three water-holding capacities were $0 \%, 26 \%$, and $52 \%$ gravel by volume. For conciseness, the water stress duration treatments are hereafter referred to by the stage at which they were imposed; the water-holding capacity treatments are referred to as the percentage of gravel incorporated.

Bloom (50\% cap fall) occurred 26 to 27 Apr. for both cultivars. The three water stress duration treatments (control excluded) were imposed on 15 May (postbloom; 19 days after full bloom), 28 June (lag phase; 63 days after full bloom), and veraison ( 5 and 11 July; 70 and 76 days after full bloom for 'Pinot noir' and 'Riesling', respectively) and continued through to harvest. Deficit imposition consisted of restricting water to $250 \mathrm{ml} /$ vine per day from the beginning of the deficit and thereafter. This volume was based on the water used by six additional 'Pinot noir' and 'Riesling' vines by determining the plants' weight loss over the 5 days (10 to 14 May) before the first water deficit was imposed. These vines were watered only on 10 May but were weighed daily and showed a mean water use of $180 \mathrm{ml} \cdot \mathrm{day}^{-1}$, with a maximum of 270 ml.day ${ }^{-1}$. We assumed that subsequent increases in vine growth would result in increased water demand; hence, we chose 250 $\mathrm{ml}$ as a reasonable volume to apply as a deficit. Daily water usage of these vines between 4 and 12 June (inclusive) was 266 and 277 ml-day ${ }^{-1}$ for 'Riesling' and 'Pinot noir', respectively, with respective maxima of 400 and $420 \mathrm{ml} \cdot \mathrm{day}^{-1}$. These results confirmed the initial assumption on appropriate water volumes to apply to deficit treatments. All irrigation water was applied manually. Vines not being subjected to water deficits were watered to runoff. The greenhouse was maintained at 26/ 20C day/night. A regular pest control program was maintained.

One fruitful shoot was retained on each vine, which was trained vertically as the experiment progressed. Shoots were topped when $1.6 \mathrm{~m}$ tall on 10 May 1991 (14 days after full bloom). Vines that failed to reach $1.6 \mathrm{~m}$ by 10 May subsequently were topped as they achieved this height. As lateral shoots grew, they were each pruned to two nodes at $\approx 15$-day intervals between mid-June and late August. Five measurements for 'Pinot noir' $(47,61$, 73, 89, and 108 days after full bloom) and six for 'Riesling' (additional measurement 125 days after full bloom) of total lateral shoot length per vine were taken from the growth removed on these pruning dates. Lateral leaf count and area, number and length of laterals, and lateral shoot internode length also were measured on each pruning date and following harvest.

Clusters per vine varied from one to three. One exposed berry per cluster per vine from the middle of the basal cluster was sampled weekly, beginning 3 to 4 days following imposition of the veraison deficit (8 July for 'Pinot noir', 15 July for 'Riesling') for berry weight and soluble solids concentration (SSC) measurements. At harvest (9 and 21 Aug. for 'Pinot noir' and 'Riesling', respectively), all clusters were collected, and cluster weight, number of berries per cluster, and berry weights 
were recorded. Also, the number and weight of shrivelled and turgid berries were recorded. Thereafter, berries were juiced, and SSC and $\mathrm{pH}$ were determined on the settled juice by an Abbé refractometer (model Mark II; AO Instruments, Buffalo, N.Y.) and a $\mathrm{pH}$ meter (Fisher Scientific, Vancouver, British Columbia, Canada), respectively. Titratable acidity (TA) was measured on 10-ml juice samples by Amerine and Ough's method (1980) using a titrator ensemble (Metrohm, Herisau, Switzerland).

Midday measurements of stomatal conductance were taken on one young, exposed, fully expanded leaf per vine following the postbloom (24 May and 12 June) and the veraison deficits (23 July and 2 Aug.), using a steady-state porometer (model LI-1600; LICOR, Lincoln, Neb.). Gathered data was used to calculate diffusive resistance and transpira- tion. Soil moisture readings also were collected from each pot before harvest on 26 July to 9 Aug. (inclusive) by time-demand refractometry (TDR) using a TDR soil moisture analyzer (Trase Soil Moisture Corp., Santa Barbara, Calif.) with 0.5-m TDR probes inserted into the pots. Two TDR readings per day were taken; one before watering $(\approx 0800$ $\mathrm{HR})$ and another $\approx 2 \mathrm{~h}$ following watering $(\approx 1030$ HR).

Data were analyzed using the SAS statistical package (SAS Institute, Cary, N.C.). Because timing of water deficits could be equated to a specific Julian day (repeated observations) or a given duration of imposed water stress (harvest measurements), the irrigation treatments were analyzed on this basis as single degree-of-freedom polynomial contrasts. Similarly, water-holding capacity treatments were numerical (percentage of gravel), so likewise these were assessed as single degree-of-freedom linear and quadratic contrasts.

\section{Results and Discussion}

Vegetative growth. As vine water stress developed, length of lateral shoot regrowth decreased, as did the number of lateral shoots. In 'Pinot noir' and 'Riesling', lateral shoot length (Table 1) decreased linearly with increased water stress duration; lag phase or veraison vines had the longest laterals and postbloom vines the shortest. However, this appeared to be an acute and largely transient response. As water stress progressed, many main leaves abscised, followed by an apparent resumption in lateral shoot growth; hence, this linear trend was reversed later in the sampling period. Lateral shoot count (Table 2) also showed the same pattern, with a marked re-

Table 1. Impact of irrigation deficit timing and soil water-holding capacity on total lateral shoot length per vine of >1.6-m-high, glasshouse-grown, 'Pinot noir' and 'Riesling' grapes.

\begin{tabular}{|c|c|c|c|c|c|c|c|c|c|c|c|}
\hline \multirow{4}{*}{$\begin{array}{l}\text { Sampling date } \\
\text { (days after } \\
\text { full bloom) }\end{array}$} & \multicolumn{11}{|c|}{ Lateral shoot length $(\mathrm{mm})$} \\
\hline & \multicolumn{6}{|c|}{ Irrigation deficit timing ${ }^{z}$} & & & & & \\
\hline & \multirow{2}{*}{$\begin{array}{l}\text { Control } \\
\text { (C) }\end{array}$} & \multirow{2}{*}{$\begin{array}{c}\text { Postbloom } \\
\text { (P) }\end{array}$} & \multirow{2}{*}{$\begin{array}{c}\text { Lag phase } \\
\text { (L) }\end{array}$} & \multirow{2}{*}{$\begin{array}{c}\text { Veraison } \\
(\mathrm{V}) \\
\end{array}$} & \multicolumn{2}{|c|}{ Significance $^{y}$} & \multicolumn{5}{|c|}{ Gravel in soil $(\%)^{\mathrm{x}}$} \\
\hline & & & & & $\mathrm{P} / \mathrm{L} / \mathrm{V}$ & C vs. others & 0 & 26 & 52 & Significance $^{y}$ & Interaction \\
\hline \multicolumn{12}{|c|}{ Pinot noir } \\
\hline $47^{w}$ & 60 & -243 & 79 & 45 & $\mathrm{~L}^{* *}$ & NS & -132 & 114 & -93 & $\mathrm{Q}^{* *}$ & NS \\
\hline 61 & 906 & 219 & 1206 & 1014 & $\mathrm{~L}^{* * * *}$ & NS & 609 & 998 & 903 & NS & NS \\
\hline 73 & 449 & 23 & 101 & 315 & $\mathrm{~L}^{* *}$ & $* * *$ & 235 & 207 & 189 & NS & NS \\
\hline 89 & 1432 & 153 & 55 & 138 & NS & $* * *$ & 589 & 543 & 201 & $\mathrm{~L}^{* *}$ & $* * *$ \\
\hline 108 & 771 & 189 & 28 & 7 & $\mathrm{~L}^{*}$ & $* * *$ & 351 & 283 & 112 & $\mathrm{~L}^{* *}$ & $* * *$ \\
\hline \multicolumn{12}{|c|}{ Riesling } \\
\hline $47^{w}$ & -112 & -434 & -233 & -175 & $\mathrm{~L}^{* * *}$ & $* *$ & -426 & -201 & -88 & $\mathrm{~L}^{* * *}$ & NS \\
\hline 61 & 388 & 75 & 300 & 385 & $\mathrm{~L}^{* * *}$ & NS & 257 & 250 & 355 & NS & NS \\
\hline 73 & 128 & 63 & 113 & 123 & NS & NS & 126 & 111 & 83 & NS & NS \\
\hline 89 & 222 & 139 & 46 & 148 & $\mathrm{Q}^{* * * *}$ & $* * *$ & 135 & 142 & 140 & NS & $*$ \\
\hline 108 & 188 & 214 & 22 & 21 & $\mathrm{~L}^{* * *}$ & $* * *$ & 138 & 118 & 78 & $\mathrm{~L}^{* *}$ & $* *$ \\
\hline 125 & 275 & 154 & 64 & 49 & $\mathrm{~L}^{* * * *}$ & $* * *$ & 142 & 141 & 124 & NS & NS \\
\hline
\end{tabular}

${ }^{2}$ Deficit imposition occurred 16, 63, and 70 ('Pinot noir') or 16, 63, and 76 ('Riesling') days after full bloom for the postbloom, lag phase, and veraison treatments, respectively.

${ }^{\mathrm{y}} \mathrm{L}=$ linear; $\mathrm{Q}=$ quadratic.

${ }^{\mathrm{x}}$ Gravel percentage inversely related to water-holding capacity.

wNegative numbers indicate that mean total lateral shoot length was $<1.6 \mathrm{~m}$ minus the height of the main shoot.

ss, *,**,**** Nonsignificant or significant at $P \leq 0.05,0.01$, or 0.001 , respectively.

Table 2. Impact of irrigation deficit timing and soil water-holding capacity on the mean number of lateral shoots per glasshouse-grown 'Pinot noir' and 'Riesling' grapevine.

\begin{tabular}{|c|c|c|c|c|c|c|c|c|c|c|c|}
\hline \multirow{4}{*}{$\begin{array}{l}\text { Sampling date } \\
\text { (days after } \\
\text { full bloom) }\end{array}$} & \multicolumn{11}{|c|}{ No. lateral shoots } \\
\hline & \multicolumn{6}{|c|}{ Irrigation deficit timing $^{2}$} & & & & & \\
\hline & \multirow{2}{*}{$\begin{array}{l}\text { Control } \\
\text { (C) }\end{array}$} & \multirow{2}{*}{$\begin{array}{c}\text { Postbloom } \\
\text { (P) }\end{array}$} & \multirow{2}{*}{$\begin{array}{c}\text { Lag phase } \\
\text { (L) }\end{array}$} & \multirow{2}{*}{$\begin{array}{c}\text { Veraison } \\
\text { (V) }\end{array}$} & \multicolumn{2}{|c|}{ Significance $^{\mathrm{y}}$} & \multicolumn{5}{|c|}{ Gravel in soil $(\%)^{\mathrm{x}}$} \\
\hline & & & & & $\mathrm{P} / \mathrm{L} / \mathrm{V}$ & C vs. others & 0 & 26 & 52 & Significance $^{y}$ & $\overline{\text { Interaction }}$ \\
\hline \multicolumn{12}{|c|}{ Pinot noir } \\
\hline 61 & 3.2 & 1.3 & 4.1 & 4.2 & $\mathrm{~L}^{* * *}$ & NS & 2.3 & 4.5 & 2.8 & $\mathrm{Q}^{* *}$ & NS \\
\hline 73 & 2.7 & 0.3 & 1.0 & 2.3 & $\mathrm{~L}^{* *}$ & $* *$ & 2.1 & 1.8 & 0.8 & $\mathrm{~L}^{* *}$ & NS \\
\hline 89 & 4.1 & 1.3 & 0.3 & 1.7 & $\mathrm{Q}^{*}$ & $* * *$ & 2.1 & 2.1 & 1.3 & NS & NS \\
\hline 108 & 3.5 & 1.2 & 0.4 & 0.1 & $\mathrm{~L}^{*}$ & $* * *$ & 1.7 & 1.4 & 0.8 & $\mathrm{~L}^{*}$ & $*$ \\
\hline \multicolumn{12}{|c|}{ Riesling } \\
\hline 61 & 3.4 & 1.1 & 2.7 & 2.2 & $\mathrm{~L}^{* *}$ & $* *$ & 1.5 & 3.0 & 2.5 & $\mathrm{Q}^{* *}$ & NS \\
\hline 73 & 3.4 & 1.0 & 1.7 & 4.2 & $\mathrm{~L}^{* * * *}, \mathrm{Q}$ & $* *$ & 3.1 & 3.1 & 1.5 & $\mathrm{~L}^{* * *}, \mathrm{Q}^{*}$ & NS \\
\hline 89 & 5.5 & 1.2 & 0.5 & 3.3 & $\mathrm{~L}^{* *}, \mathrm{Q}^{* * *}$ & $* * *$ & 3.0 & 3.0 & 1.8 & $\mathrm{~L}^{* *}$ & $* *$ \\
\hline 108 & 3.8 & 1.6 & 0.8 & 0.2 & $\mathrm{~L}^{* *}$ & $* * *$ & 2.0 & 1.9 & 0.9 & $\mathrm{~L}^{* *}$ & $* * *$ \\
\hline 125 & 5.2 & 2.2 & 0.4 & 0.6 & $\mathrm{~L}^{* * *}$ & $* * *$ & 2.4 & 2.3 & 1.5 & $\mathrm{~L}^{* *}$ & $*$ \\
\hline
\end{tabular}

${ }^{2}$ Deficit imposition occurred 16, 63, and 70 ('Pinot noir') or 16, 63, and 76 ('Riesling') days after full bloom for the postbloom, lag phase, and veraison treatments, respectively.

${ }^{\mathrm{y}} \mathrm{L}=$ linear; $\mathrm{Q}=$ quadratic.

${ }^{\mathrm{x}}$ Gravel percentage inversely related to water-holding capacity.

ns, *,**,*** Nonsignificant or significant at $P \leq 0.05,0.01$, or 0.001 , respectively. 
duction occurring immediately after imposing each deficit, followed by an increase in subsequent sampling dates. Control vines had more laterals and longer regrowth than the deficit treatments (considered as one group) for both cultivars on most sampling dates (Tables 1 and 2). Cumulative lateral shoot length (Table 3) showed a linear decrease with increased water stress duration, and the control differed from the deficit treatments on three of five sampling dates for 'Pinot noir' and all six sampling dates for 'Riesling'. With two exceptions, lateral leaf area per vine and per leaf and lateral leaf count (Table 4) were reduced linearly with water stress duration in both cultivars. The deficit treatments also displayed less lateral shoot growth than the control. These results are consistent with prior studies (Matthews et al., 1987; Peacock et al., 1987; Schultz and Matthews, 1988a; Smart and Coombe, 1983), which indicated that shoot growth in fieldgrown grapevines is sensitive to early season, continual, water deficits. However, the resumption in lateral shoot regrowth after 40 to 50 days, especially in the postbloom treatments, had not been documented for grapevines.

Lateral shoot growth (Table 1) and lateral shoot count (Table 2) of 'Riesling' vines were reduced linearly on some sampling dates with decreasing water-holding capacity of the soil. Cumulative lateral shoot growth (Table 3), however, increased with decreasing waterholding capacity on the first two sampling dates. 'Pinot noir' vines showed less of a response in lateral shoot count and cumulative lateral shoot growth; in most cases, the trends were quadratic, with $26 \%$ gravel yielding the highest values. Lateral leaf area response to increasing soil gravel content was also quadratic in nature wherein the $26 \%$ gravel resulted in the largest leaf area (Table 4), although lateral leaf size with $52 \%$ gravel was less than with $0 \%$. This relationship was presumably due to an improvement in soil aeration with $26 \%$ gravel, whereas $52 \%$ reduced water-holding capacity and, hence, increased potential for water stress.

Table 3. Impact of irrigation deficit timing and soil water-holding capacity on cumulative lateral shoot length of glasshouse-grown 'Pinot noir' and 'Riesling' grapes.

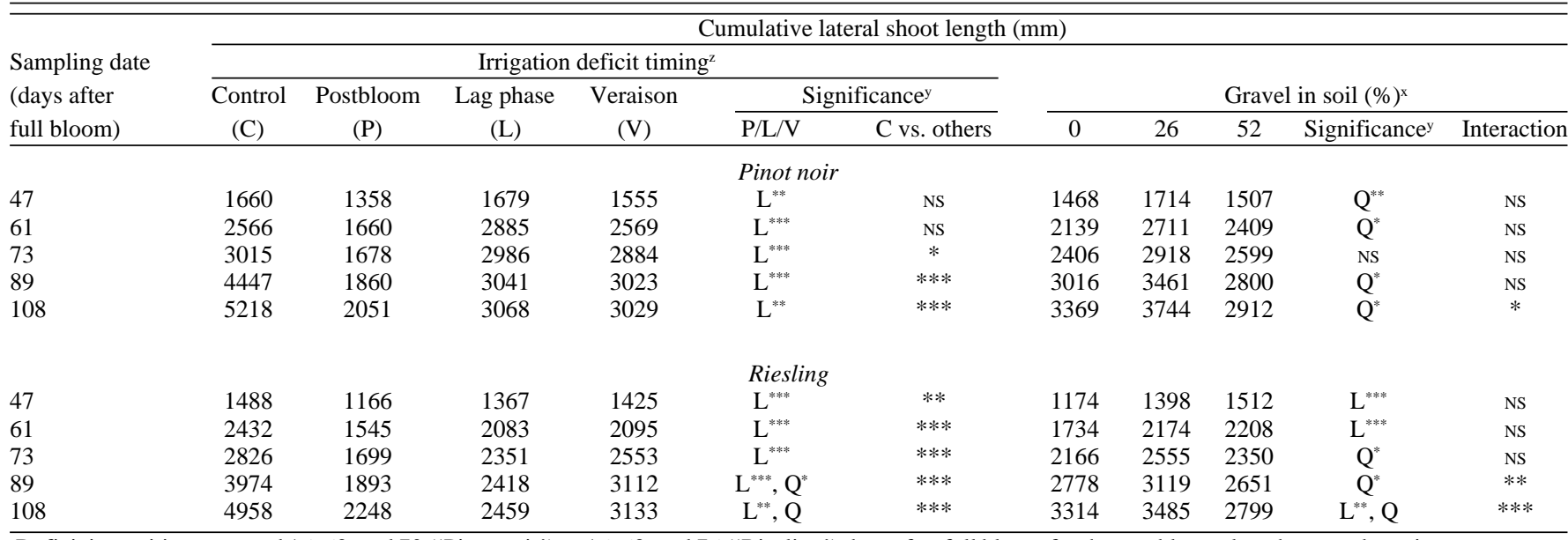

${ }^{2}$ Deficit imposition occurred 16, 63, and 70 ('Pinot noir') or 16, 63, and 76 ('Riesling') days after full bloom for the postbloom, lag phase, and veraison treatments, respectively.

${ }^{y} \mathrm{~L}=$ linear; $\mathrm{Q}=$ quadratic.

${ }^{\mathrm{x}}$ Gravel percentage inversely related to water-holding capacity.

ws, ${ }^{*}, * *, * * *$ Nonsignificant or significant at $P \leq 0.05,0.01$, or 0.001 , respectively.

Table 4. Impact of irrigation deficit timing and soil water-holding capacity on leaf area, yield, and berry composition of glasshouse-grown 'Pinot noir' and 'Riesling' grapes at harvest.

\begin{tabular}{|c|c|c|c|c|c|c|c|c|c|c|}
\hline \multirow[b]{3}{*}{ Variable } & \multicolumn{6}{|c|}{ Irrigation deficit timing ${ }^{\mathrm{z}}$} & & & & \\
\hline & \multirow{2}{*}{$\begin{array}{c}\text { Control } \\
(\mathrm{C})\end{array}$} & \multirow{2}{*}{$\begin{array}{c}\text { Postbloom } \\
(\mathrm{P})\end{array}$} & \multirow{2}{*}{$\begin{array}{c}\text { Lag phase } \\
\text { (L) }\end{array}$} & \multirow{2}{*}{$\begin{array}{c}\text { Veraison } \\
\text { (V) }\end{array}$} & \multicolumn{2}{|c|}{ Significance ${ }^{y}$} & \multicolumn{4}{|c|}{ Gravel in soil $(\%)^{\mathrm{x}}$} \\
\hline & & & & & $\mathrm{P} / \mathrm{L} / \mathrm{V}$ & C vs. others & 0 & 26 & 52 & Significance $^{y}$ \\
\hline \multicolumn{11}{|c|}{ Pinot noir } \\
\hline Lateral leaf area $\left(\mathrm{cm}^{2}\right)$ & 1609 & 509 & 772 & 974 & $\mathrm{~L}^{* *}$ & $* * *$ & 936 & 1208 & 755 & $Q^{* * *}$ \\
\hline No. lateral leaves/vine & 47 & 21 & 30 & 29 & NS & $* * *$ & 33 & 35 & 26 & NS \\
\hline Mean area/leaf $\left(\mathrm{cm}^{2}\right)$ & 34 & 24 & 25 & 34 & $\mathrm{~L}^{* *}, \mathrm{Q}$ & $*$ & 27 & 33 & 28 & $\mathrm{Q}^{*}$ \\
\hline Cluster wt (g) & 15.5 & 19.1 & 23.4 & 24.8 & NS & $*$ & 21.4 & 24.1 & 16.5 & NS \\
\hline Berry wt (g) & 0.97 & 0.71 & 0.80 & 0.95 & $\mathrm{~L}^{* *}$ & $* *$ & 0.91 & 0.84 & 0.82 & NS \\
\hline Shrivelled berries (\%) & 9.5 & 49.6 & 38.0 & 21.5 & $\mathrm{~L}^{* *}$ & **** & 23.1 & 34.4 & 31.4 & NS \\
\hline Soluble solids concentration & 24.4 & 27.3 & 26.6 & 25.7 & $\mathrm{~L}^{*}$ & $* * *$ & 25.7 & 25.8 & 26.4 & NS \\
\hline Titratable acidity $\left(\mathrm{g} \cdot \mathrm{liter}^{-1}\right)$ & 8.1 & 8.5 & 8.3 & 8.3 & NS & NS & 8.3 & 8.4 & 8.1 & NS \\
\hline $\mathrm{pH}$ & 3.69 & 3.82 & 3.85 & 3.81 & NS & $* * *$ & 3.78 & 3.79 & 3.81 & NS \\
\hline \multicolumn{11}{|c|}{ Riesling ${ }^{w}$} \\
\hline Lateral leaf area $\left(\mathrm{cm}^{2}\right)$ & 2447 & 590 & 971 & 1421 & $\mathrm{~L}^{* * *}, \mathrm{Q}^{*}$ & $* * *$ & 1443 & 1594 & 1036 & $\mathrm{~L}^{* *}, \mathrm{Q}$ \\
\hline No. lateral leaves/vine & 67 & 22 & 33 & 38 & $\mathrm{~L}^{* * *}$ & $* * *$ & 42 & 42 & 37 & NS \\
\hline Mean area/leaf & 35 & 29 & 29 & 37 & $\mathrm{Q}^{* *}$ & NS & 32 & 38 & 27 & $\mathrm{Q}^{* * * *}$ \\
\hline Cluster wt (g) & 14.2 & 13.9 & 9.9 & 13.1 & NS & NS & 13.2 & 15.9 & 9.3 & NS \\
\hline Berry wt $(\mathrm{g})$ & 0.62 & 0.56 & 0.61 & 0.65 & $\mathrm{~L}^{*}$ & NS & 0.63 & 0.62 & 0.58 & NS \\
\hline Shrivelled berries (\%) & 17.6 & 18.5 & 16.3 & 19.0 & NS & NS & 15.9 & 18.3 & 19.6 & NS \\
\hline SSC & 21.1 & 21.9 & 22.9 & 23.0 & $\mathrm{~L}^{* *}$ & $* *$ & 21.8 & 22.1 & 22.7 & NS \\
\hline TA $\left(\mathrm{g} \cdot\right.$ liter-1 $\left.^{-1}\right)$ & 9.3 & 9.5 & 9.4 & 8.9 & NS & NS & 9.3 & 9.4 & 9.0 & NS \\
\hline $\mathrm{pH}$ & 3.44 & 3.42 & 3.52 & 3.53 & $\mathrm{~L}^{* * * *}$ & $* * *$ & 3.50 & 3.49 & 3.45 & NS \\
\hline
\end{tabular}

${ }^{2}$ Deficit imposition occurred 16, 63, and 70 ('Pinot noir') or 16, 63, and 76 ('Riesling') days after full bloom for the postbloom, lag phase, and veraison treatments, respectively.

${ }^{\mathrm{y}} \mathrm{L}=$ linear; $\mathrm{Q}=$ quadratic.

${ }^{x}$ Gravel percentage inversely related to water-holding capacity.

wIrrigation $\times$ water-holding capacity interactions were present for lateral leaf area and lateral leaf count, both at $P \leq 0.001$.

Ns, ${ }^{*, * *, * * * *}$ Nonsignificant or significant at $P \leq 0.05,0.01$, or 0.001 , respectively. 
Water stress duration $\times$ water-holding capacity interactions occurred on the fourth sampling dates and thereafter in both cultivars for lateral shoot length, lateral shoot count, and cumulative lateral shoot length (Tables 1-3). These interactions showed that linear reductions in lateral shoot length, lateral shoot count, and cumulative lateral shoot length with respect to decreased water-holding capacity occurred chiefly in the control vines, with a lesser response in the other water deficit treatments (data not shown). The result was the same for lateral leaf area per vine in 'Riesling' (Table 4). These data suggest that soil waterholding capacity may not provide an additional significant limitation to vine growth in the presence of imposed irrigation deficits.

Yield components. By imposing water deficits, berry weight was reduced linearly in both cultivars on some sampling dates. 'Pinot noir' berries displayed linear berry weight decreases with increased water stress duration on the first and fifth sampling dates; 'Riesling' berry weight responded in a similar fashion on the third and final sampling dates (Table 5). Water stress duration linearly reduced berry weight of both cultivars at harvest (Table 4). This result seemed to be a function of a linear increase in the number of shrivelled berries in 'Pinot noir' with increasing water stress duration. Although cluster count per vine was variable, yield per vine (data not shown), cluster weight (Table 4), and berries per cluster (data not shown) were not influenced in either cultivar by deficit timing; however, 'Pinot noir' control vines produced lighter clusters than those of the water deficit treatments. The berry weight reductions found in this study are consistent with trends reported for field-grown and potted 'Cabernet franc' (Hardie and Considine, 1976; Matthews and Anderson, 1989; Matthews et al., 1987) for which early irrigation deficits were particularly effective in reducing berry weight.

Berry weight increased linearly with decreasing water-holding capacity on all five sampling dates for 'Riesling' but on only one of five for 'Pinot noir' (Table 5). These berry weight increases may have been a response to fewer lateral shoots, leading to an increased carbohydrate allocation to the berries. Reynolds (1988) provided evidence of an increased berry weight and lateral shoot reduction relationship resulting from $\beta$-[(4-chlorophenyl)methyl]- $\alpha$ (1,1-dimethylethyl)-1- $H$-1,2,4-triazole-1-ethanol (paclobutrazol) applications to field-grown 'Riesling'. There was no effect of water-holding capacity on berry weight at harvest for either cultivar in our study (Table 4); however, the percentage of shrivelled berries tended to increase linearly with decreasing water-holding capacity (Table 4). Neither cluster weight (Table 4) nor berries per cluster (data not shown) for either cultivar were affected by soil water-holding capacity.

Berry composition. Water stress also affected juice composition. The response of SSC was predominantly linear with respect to water stress duration in 'Pinot noir' (Table 6), with postbloom and lag phase treatments having the highest values. The controls had lower SSC than plants with water deficit toward the

Table 5. Impact of irrigation deficit timing and soil water-holding capacity on berry weight of glasshouse-grown 'Pinot noir' and 'Riesling' grapes.

\begin{tabular}{|c|c|c|c|c|c|c|c|c|c|c|c|}
\hline \multirow{4}{*}{$\begin{array}{l}\text { Sampling date } \\
\text { (days after } \\
\text { full bloom) }\end{array}$} & \multicolumn{11}{|c|}{ Berry wt $(\mathrm{g})$} \\
\hline & \multirow{3}{*}{$\begin{array}{l}\text { Control } \\
\text { (C) }\end{array}$} & \multirow{3}{*}{$\begin{array}{l}\text { Postbloom } \\
\text { (P) }\end{array}$} & \multicolumn{4}{|c|}{ Irrigation deficit timing ${ }^{2}$} & \multirow[b]{3}{*}{0} & \multirow[b]{3}{*}{26} & \multirow[b]{3}{*}{52} & \multirow{2}{*}{\multicolumn{2}{|c|}{ Gravel in soil $(\%)^{x}$}} \\
\hline & & & \multirow{2}{*}{$\begin{array}{c}\text { Lag phase } \\
\text { (L) }\end{array}$} & \multirow{2}{*}{$\begin{array}{c}\text { Veraison } \\
\text { (V) }\end{array}$} & \multicolumn{2}{|c|}{ Significance } & & & & & \\
\hline & & & & & $\mathrm{P} / \mathrm{L} / \mathrm{V}$ & C vs. others & & & & Significance $^{y}$ & Interaction \\
\hline & & & & & \multicolumn{2}{|c|}{ Pinot noir } & & & & & \\
\hline 73 & 0.97 & 0.92 & 1.07 & 1.15 & $\mathrm{~L}^{*}$ & NS & 0.89 & 1.05 & 1.13 & $\mathrm{~L}^{*}$ & NS \\
\hline 80 & 1.03 & 0.94 & 0.99 & 1.09 & NS & NS & 0.99 & 1.01 & 1.03 & NS & NS \\
\hline 88 & 1.09 & 1.04 & 1.08 & 1.14 & NS & NS & 1.06 & 1.16 & 1.05 & NS & NS \\
\hline 94 & 1.19 & 1.01 & 1.04 & 1.15 & NS & NS & 1.10 & 1.15 & 1.04 & NS & NS \\
\hline \multirow[t]{2}{*}{102} & 1.06 & 0.79 & 1.00 & 1.24 & $\mathrm{~L}^{* *}$ & NS & 1.10 & 0.99 & 0.98 & NS & NS \\
\hline & & & & & \multicolumn{2}{|c|}{ Riesling } & & & & & \\
\hline 80 & 0.80 & 0.76 & 0.77 & 0.70 & NS & NS & 0.68 & 0.75 & 0.83 & $\mathrm{~L}^{*}$ & NS \\
\hline 89 & 1.02 & 0.94 & 0.99 & 1.02 & NS & NS & 0.91 & 0.98 & 1.08 & $\mathrm{~L}^{*}$ & NS \\
\hline 97 & 0.99 & 0.82 & 0.91 & 0.96 & $\mathrm{~L}^{*}$ & $*$ & 0.87 & 0.89 & 0.99 & $\mathrm{~L}^{*}$ & $*$ \\
\hline 104 & 1.00 & 0.91 & 0.89 & 0.96 & NS & NS & 0.84 & 0.95 & 1.03 & $\mathrm{~L}^{*}$ & NS \\
\hline 111 & 1.14 & 0.87 & 1.00 & 1.03 & $\mathrm{~L}^{*}$ & $*$ & 0.90 & 1.03 & 1.07 & $\mathrm{~L}^{*}$ & NS \\
\hline
\end{tabular}

${ }^{2}$ Deficit imposition occurred 16, 63, and 70 ('Pinot noir') or 16, 63, and 76 ('Riesling') days after full bloom for the postbloom, lag phase, and veraison treatments, respectively.

${ }^{\mathrm{y}} \mathrm{L}=$ linear; $\mathrm{Q}=$ quadratic.

${ }^{\mathrm{x}}$ Gravel percentage inversely related to water-holding capacity.

ns, *** Nonsignificant or significant at $P \leq 0.05$ or 0.01 , respectively.

Table 6. Impact of irrigation deficit timing and soil water-holding capacity on soluble solids concentration (SSC) of glasshouse-grown 'Pinot noir' and 'Riesling' grapes.

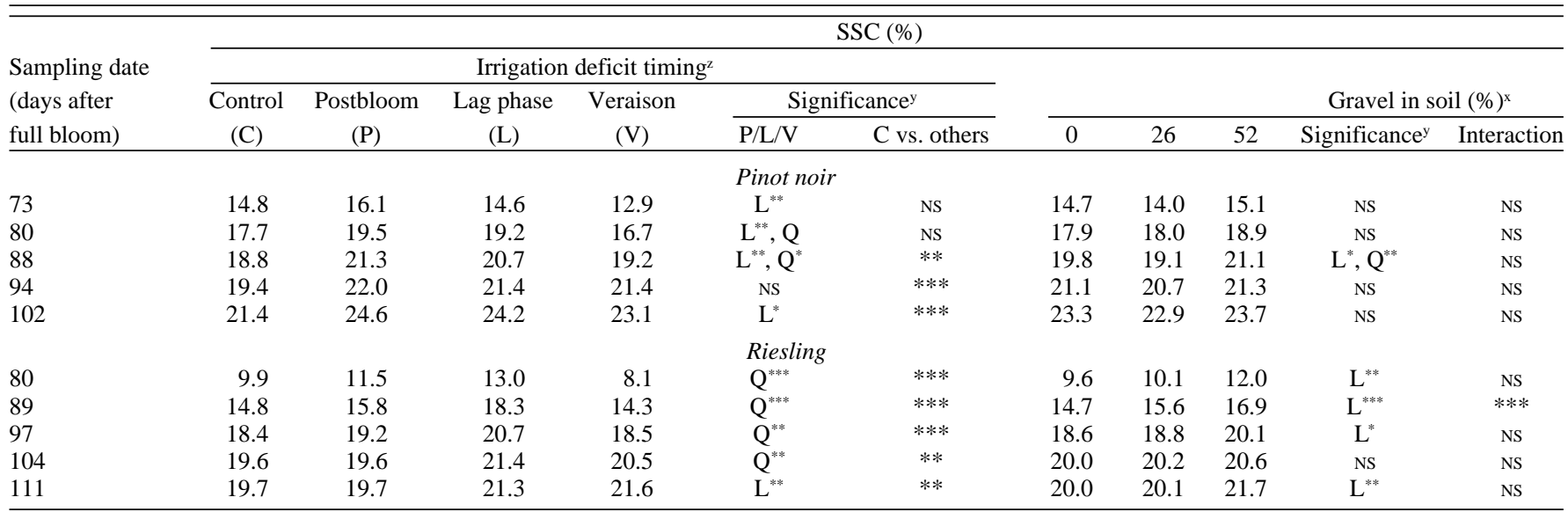

${ }^{2}$ Deficit imposition occurred 16, 63, and 70 ('Pinot noir') or 16, 63, and 76 ('Riesling') days after full bloom for the postbloom, lag phase, and veraison treatments, respectively.

${ }^{y} \mathrm{~L}=$ linear; $\mathrm{Q}=$ quadratic.

${ }^{\mathrm{x}}$ Gravel percentage inversely related to water-holding capacity.

Ns, $, * * *, * * *$ Nonsignificant or significant at $P \leq 0.05,0.01$, or 0.001 , respectively. 
Table. 7. Impact of irrigation deficit timing and soil water-holding capacity on transpiration of glasshouse-grown 'Pinot noir' and 'Riesling' grapes.

Transpiration $\left(\mu \mathrm{g} \mathrm{H} \mathrm{H}_{2} \mathrm{O} / \mathrm{cm}^{-1}\right)$

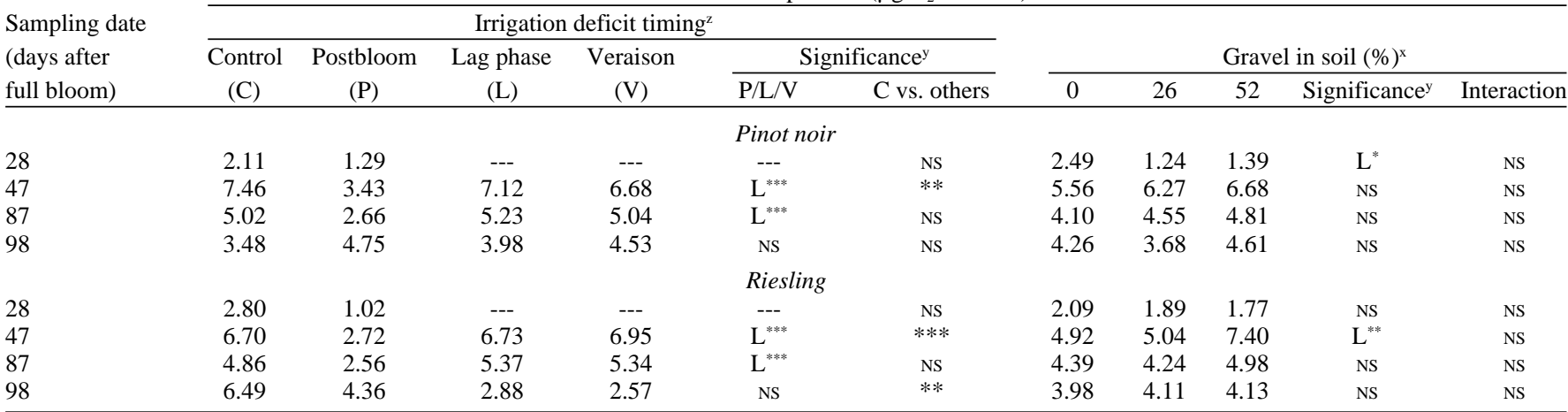

${ }^{2}$ Deficit imposition occurred 16, 63, and 70 ('Pinot noir') or 16, 63, and 76 ('Riesling') days after full bloom for the postbloom, lag phase, and veraison treatments, respectively.

${ }^{\mathrm{y}} \mathrm{L}=$ linear; $\mathrm{Q}=$ quadratic

${ }^{\mathrm{x}}$ Gravel percentage inversely related to water-holding capacity.

Ns, ${ }^{*}, * *, * * *$ Nonsignificant or significant at $P \leq 0.05,0.01$, or 0.001 , respectively.

latter stages of berry maturation. In 'Riesling', SSC was highest in the lag-phase fruit throughout most of berry maturation and lowest in postbloom and veraison fruit; on all sampling dates, the control plants had lower SSC than those exposed to water deficit. In the latter stages of berry maturation, SSC for the veraison treatment increased above the control in both cultivars. This relationship may be due to berry desiccation or may be attributed to the reduction in lateral shoot growth in the veraison treatment, with a concomitant reallocation of carbohydrates to the fruit. Among others, Reynolds et al. (1991) have demonstrated inverse relationships between grape berry SSC and lateral shoot growth. SSC and $\mathrm{pH}$ at harvest also increased linearly with increasing water stress duration, likely as a result of a concentration effect due to reduced berry size (skin area : volume ratio) and to berry shrivelling (Table 4). There was no effect of water stress duration on TA (Table 4). These results are mostly in agreement with those of Matthews and Anderson (1988), who found higher SSC, $\mathrm{pH}$, and anthocyanins in field-grown 'Cabernet franc' berries subjected to early water deficits. They also are consistent with Hardie and Considine (1976), who found increases in SSC, $\mathrm{pH}$, and skin color of potted 'Cabernet franc' as water stress duration increased.

SSC was enhanced in both cultivars (Table 6) by decreasing water-holding capacity, and the effects were predominantly linear. However, as with lateral shoot growth and berry weight, SSC of 'Pinot noir' was influenced less than that of 'Riesling' by soil waterholding capacity: 'Riesling' showed linear increases in SSC with decreasing water-holding capacity on four of five sampling dates, compared to only one of five sampling dates for 'Pinot noir'. Because berry weight and SSC increased with decreasing water-holding capacity, the concomitant reductions in lateral shoot growth (i.e., carbohydrate reallocation) likely may have contributed to this response. SSC (both cultivars) and pH ('Pinot noir' only) at harvest tended to increase linearly in response to lowering water-holding capacity, albeit not significantly. TA also was not strongly affected (Table 4). The increased percentage of shrivelled berries partly may have accounted for the increase in SSC and $\mathrm{pH}$ at harvest and also may have militated against statistically significant trends in the data.

Water relations. Stomatal resistance is known to increase significantly when vines experience severe water stress (Smart and Coombe, 1983). In this trial, stomatal conductance (data not shown) and transpiration of 'Pinot noir' and 'Riesling' (Table 7) decreased linearly with increasing water stress duration on two of four sampling dates, although the response to control, lag phase, and veraison treatments differed little from each other. Transpiration decreased markedly in the lag phase and veraison treatments between the third and final sampling dates ( 87 and 98 days after full bloom, respectively), reflecting a rapid response to these water deficits applied 63 and 76 days following full bloom, respectively. Control vines had the highest transpiration rates on one of four dates for 'Pinot noir' and on two of four dates for 'Riesling' (Table 7). Correspondingly, stomatal resistance was highest in the postbloom treatments (data not shown). Smart (1974) demonstrated large decreases in transpiration rate, hence higher stomatal resistance, and leaf water potential of water-stressed, field-grown, 'Shiraz' grapevines. Grimes and Williams (1990) also associated yield reductions in drought-stressed 'Thompson Seedless' vines with increased stomatal resistance. Schultz and Matthews (1988b) suggested that, as water deficit duration increases, vapor filling of xylem vessels (i.e., water cavitation within) gradually leads to increased hydraulic resistance. Hence, in our experiment, despite continually applying small volumes of water to the stressed vines, stomatal resistance gradually increased as the plants grew.

In 'Pinot noir', stomatal conductance (data not shown) and transpiration (Table 7) decreased linearly on one of four sampling dates with decreasing soil water-holding capacity. Stomatal resistance (data not shown) increased linearly on two of four sampling dates with increasing water-holding capacity. 'Riesling' water relations (Table 7) were similarly and more consistently affected by water-holding capacity than 'Pinot noir' in terms of growth, yield, and fruit composition. Lack of water stress duration $\times$ water-holding capacity interactions for water relations suggests that reducing soil water-holding capacity did not necessarily increase the degree of physiological water stress imposed by the various irrigation deficits.

Soil moisture readings, began 72,28 , and 21 days following imposition of the irrigation deficits, indicated a primarily quadratic relationship for 'Pinot noir' in which the veraison deficit produced the lowest soil moisture content (Table 8). In the case of 'Riesling', the relationship was linear, wherein soil moisture decreased as water stress duration decreased (Table 8). These observations can be explained by the increased lateral shoot leaf areas, hence increased water demand, with decreasing water stress duration.

Table 8. Soil moisture (percent by volume) in response to four irrigation deficit treatments and three water-holding capacities for glasshousegrown 'Pinot noir' and 'Riesling' grapes, 26 July to 9 Aug. 1991.

\begin{tabular}{|c|c|c|}
\hline \multirow[b]{3}{*}{ Factor } & \multicolumn{2}{|c|}{ Soil moisture (\%) } \\
\hline & \multicolumn{2}{|c|}{ Cultivar } \\
\hline & Pinot noir & Rieslin \\
\hline \multicolumn{3}{|c|}{ Irrigation deficit time } \\
\hline Postbloom $(86)^{z}$ & 9.2 & 9.7 \\
\hline Lag phase (42) & 9.2 & 8.9 \\
\hline Veraison (33) & 7.7 & 7.8 \\
\hline Control (C) & 21.4 & 23.6 \\
\hline Significance $y$ & $\mathrm{~L}^{* * *}, \mathrm{Q}$ & $\mathrm{L}^{* * *}$ \\
\hline C vs. others & $* * *$ & $* * *$ \\
\hline
\end{tabular}

$\begin{array}{lrr}\text { Gravel in soil }(\%)^{\mathrm{x}} & & \\ 0 & 13.8 & 17.2 \\ 26 & 11.9 & 11.4 \\ 52 & 9.9 & 8.9 \\ \text { Significance }^{\mathrm{y}} & \mathrm{L}^{* * *}, \mathrm{Q} & \mathrm{L}^{* * *}, \mathrm{Q} \\ \text { Interaction }^{* * *} & * * *\end{array}$

${ }^{2}$ Water stress duration in days.

${ }^{\prime} \mathrm{L}=$ linear; $\mathrm{Q}=$ quadratic.

${ }^{\mathrm{x}}$ Gravel percentage inversely related to water-holding capacity.

${ }^{* * *}$ Significant at $P \leq 0.001$. 
Soil water content decreased linearly with decreasing water-holding capacity in both cultivars (Table 8). The water stress duration $\times$ water-holding capacity interactions showed a greater response to water-holding capacity in the control and veraison deficit vines, with veraison-52\% gravel treatments resulting in the lowest soil water volumes $(6.9 \%$ and $6.0 \%$ for 'Pinot noir' and 'Riesling', respectively).

Growth, yield, and juice composition of glasshouse-grown grapes were affected by water stress duration and soil water-holding capacity. Although both cultivars displayed similar responses to water stress duration, 'Pinot noir' appeared to be less affected than 'Riesling' to reduced water-holding capacity. This difference has important implications as the wine industry in British Columbia expands into the southern portion of the Okanagan Valley, where rainfall is scarce and soil waterholding capacity is low. Results from this glasshouse trial suggest that these sites for potential expansion may not be suitable for 'Riesling', but 'Pinot noir' and related cultivars probably would be well adapted.

Observations of soil moisture levels in response to water stress duration suggest that deficits at veraison reduce soil water to lower levels than do earlier continual deficits due to greater water demands (from more leaf area) of the previously unstressed vines. This situation is exacerbated in low water-holding capacity soils, in which soil water content may fall to low levels following veraison-imposed irrigation deficits. If veraison deficits are anticipated, a grower must realize that water requirements may be greater than for a previously stressed vine containing less leaf area (hence transpirational surface), and volume or frequency of irrigation water should be adjusted accordingly.

\section{Literature Cited}

Amerine, M.A. and C.S. Ough. 1980. Methods for the analysis of musts and wines. Wiley, New York.

Grimes, D.W. and L.E. Williams. 1990. Irrigation effects on plant water relations and productivity of Thompson Seedless grapevines. Crop Sci. 30:255-260.

Hardie, W.J. and J.A. Considine. 1976. Response of grapes to water-deficit stress in particular stages of development. Amer. J. Enol. Viticu lt. 27:5561.

Matthews, M.A. and M.M. Anderson. 1988. Fruit ripening in Vitis vinifera L.: Responses to seasonal water deficits. Amer. J. Enol. Viticult. 39:313-320.

Matthews, M.A. and M.M. Anderson. 1989. Reproductive development in grape (Vitis vinifera $\mathrm{L}$.) Responses to seasonal water deficits. Amer. J. Enol. Viticult. 40:52-60.

Matthews, M.A., M.M. Anderson, and H.R. Schultz. 1987. Phenological and growth responses to early and late season water deficits in Cabernet franc. Vitis 26:147-160.

Naylor, A.P., A.G. Reynolds, P. Parchomchuk, R. Berard, and E.J. Hogue. 1992. Impact of irrigation deficit timing and vineyard floor management on vine performance and water relations of young Vitis vinifera cultivar Gewürztraminer vines. Amer. J. Enol. Viticult. 43:401. (Abstr.)

Peacock, W.L., L.P. Christensen, and H.L. Andris. 1987. Development of a drip irrigation schedule for average-canopy vineyards in the San Joaquin Valley. Amer. J. Enol. Viticult. 38:113-119.

Reynolds, A.G. 1988. Inhibition of lateral shoot growth in summer-hedged 'Riesling' grapevines by paclobutrazol. HortScience 23:728-730.

Reynolds, A.G., D.A. Wardle, A.C. Cottrell, and A.P. Gaunce. 1991. Advancement of 'Riesling' fruit maturity by paclobutrazol-induced reduction of lateral shoot growth. J. Amer. Soc. Hort. Sci. 117:430-435.

Schultz, H.R. and M.M. Matthews. 1988a. Vegetative growth distribution during water deficits in Vitis vinifera L. Austral. J. Plant Physiol. 15:641656.

Schultz, H.R. and M.M. Matthews. 1988b. Resistance to water transport in shoots of Vitis vinifera L. Relation to growth at low water potential. Plant Physiol. 88:718-724.

Smart, R.E. 1974. Aspects of water relations of the grapevine. Amer. J. Enol. Viticult. 25:84-91.

Smart, R.E. and B.G. Coombe. 1983. Water relations of grapevines, p. 137-196. In: T.T. Kozlowski (ed.). Water deficits and plant growth. vol. VII. Academic, New York.

Smart, R.E., J.B. Robinson, G. Due, and C.J. Brien. 1985. Canopy microclimate modification for the cultivar Shiraz. II. Effects on must and wine composition. Vitis 24:119-128.

Trought, M.C.T. and A.P. Naylor. 1988. Irrigation responses in a cool climate, p. 156-160. In: R.E. Smart, R.J. Thornton, S.B. Rodriguez, and J.E. Young (eds.). Proc. 2nd Intl. Symp. Cool Climate Viticult. Oenol. N.Z. Soc. Viticult. and Oenol., Auckland. 